
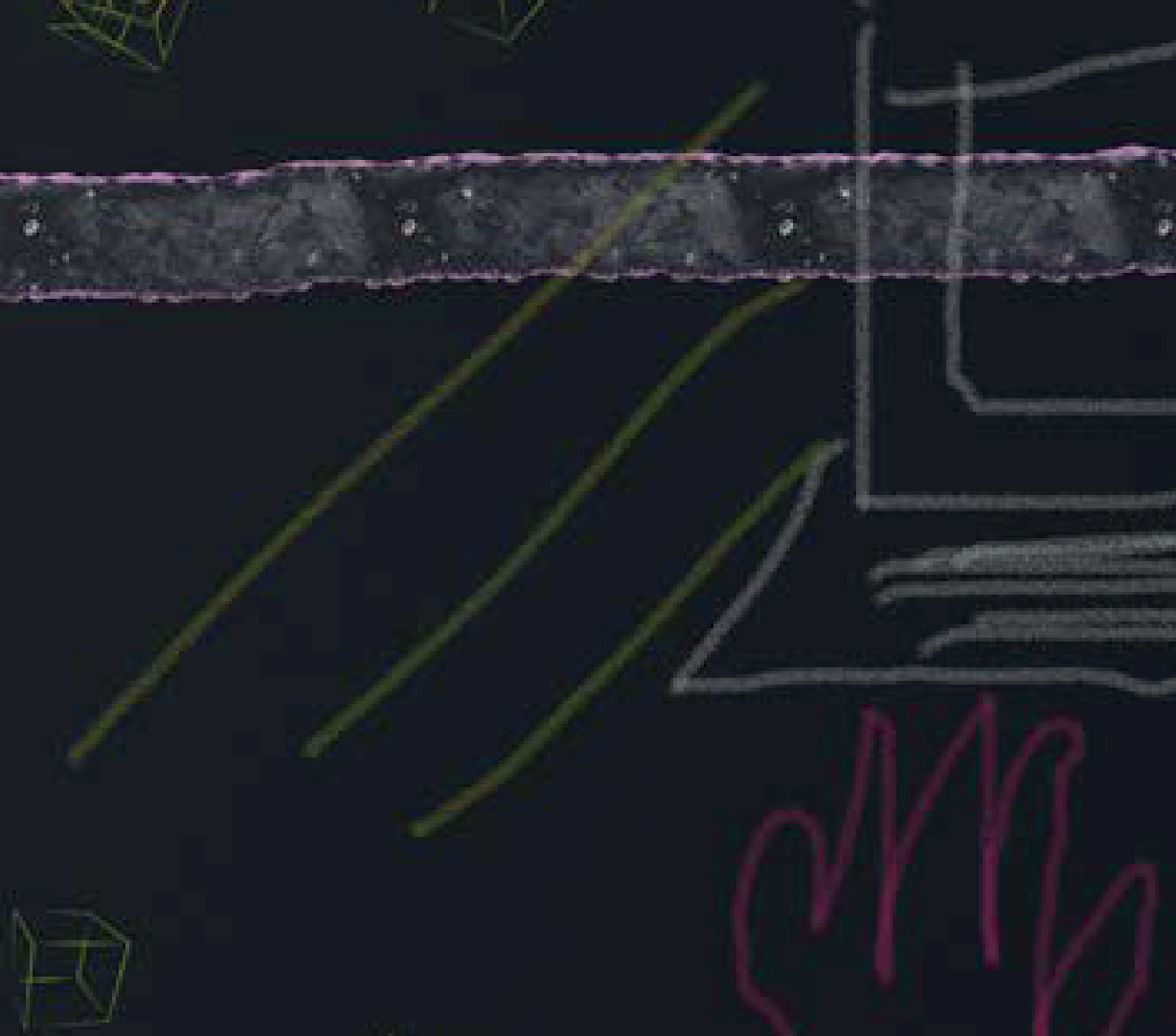


\section{EL COVID-19 Y EL CONSULTORIO PSICO- LÓGICO EN LÍNEA. ELEMENTOS BÁSICOS PARA BRINDAR TERAPIA PSICOLÓGICA A DISTANCIA}

\section{EL COVID-19 AND THE ONLINE PSYCHO- LOGICAL OFFICE. BASIC ELEMENTS TO PROVIDE DISTANCE PSYCHOLOGICAL THERAPY}

\section{Graciela Gordillo Castillo}

http://doi.org/10.37646/huella.v15i15.530

Notas sobre la autora:

Psicóloga. Psicoterapeuta, Especialista y Maestra en Docencia para la Educación Media Superior y Superior. Docente en la Universidad La Salle Pachuca.

Esta investigación fue financiada con recursos de la autora. La autora no tiene ningún conflicto de interés al haber hecho esta investigación..

Remita cualquier duda sobre este artículo al siguiente correo electrónico: graciela.gordillo@lasallep.mx

Recibido: 14/01/2021 Corregido: 26/03/2021 Aceptado:1/06/2021 


\section{Resumen}

Seguir atendiendo la consulta psicológica durante el confinamiento por la pandemia de COVID-19, hizo necesaria la utilización de las herramientas digitales de videollamada. La importancia de seguir atendiendo los lineamientos que conforman una atención psicológica adecuada, en modalidad a distancia, conlleva a indicar los elementos básicos que se ven involucrados en la psicoterapia en línea.

Palabras clave: Psicología, terapia en línea, consultorio psicológico en línea, lineamientos, normatividad.

\section{Abstract}

Continuing to attend the psychological consultation during confinement due to the COVID-19 pandemic, made necessary the use of digital video-call tools. The importance of maintaining the guidelines that make up adequate psychological care, in distance mode, leads to indicate the basic elements that are involved in online psychotherapy.

Keywords: Psychology, online therapy, online psychological clinic, guidelines, normativity. 


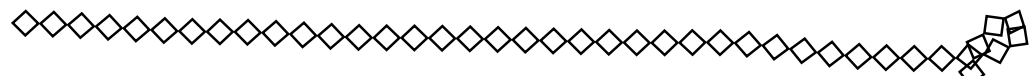

\section{INTRODUCCIÓN}

El propósito del presente trabajo es proporcionar información apegada a los lineamientos que indican las Normas Mexicanas de Salud, la Ley Federal del Manejo de la Información y Protección de Datos Personales, las directrices para la práctica de la psicología a distancia sugeridas por la Asociación Americana de Psicología, así como las condiciones materiales para su realización. Debido a la importancia que actualmente significa recibir atención psicológica a través de plataformas o aplicaciones en línea. Aspira el presente servir como un instrumento de referencia que recopile de manera práctica los elementos básicos con que debe llevarse a cabo la psicoterapia en línea, en México, a partir de la pandemia por COVID-19 y brindar una apropiada intervención a distancia.

\section{LA PSICOTERAPIA EN LÍNEA}

La pandemia por COVID-19 modificó las prácticas regulares en la consulta psicológica. La orden de confinamiento provocó que se cerraran gran cantidad de espacios laborales, entre ellos los consultorios psicológicos, debido a las restricciones sanitarias estipuladas como medida preventiva para evitar contagios en espacios cerrados. Actualmente, México se encuentra en el proceso de reapertura escalonada y ordenada de las actividades económicas. Se prevé la reapertura de consultorios hasta que los lineamientos del semáforo de riesgo epidemiológico se encuentren en color naranja (Secretaría de Salud, 2020); lo que ha significado, como consecuencia, el aumento en la oferta de la psicoterapia en línea.

Aunque ya existían la orientación y la consejería psicológicas en línea -y como sus propias definiciones lo precisan-, estas no significan recibir psicoterapia, por lo que fue muy evidente la necesidad del traslado del consultorio psicológico a la atención a distancia.

Inmediatamente después del confinamiento importantes universidades en nuestro país pusieron a disposición de los usuarios, espacios de atención psicológica de manera telefónica, primeramente, dirigido al personal médico y de la salud que atendía la emergencia por COVID-19, posteriormente para el público en general. Es el ejemplo dado de la Universidad Nacional Autónoma de México (UNAM), que brinda 
el acceso a través de una dirección electrónica ubicada en su plataforma, misma que está avalada por la Secretaría de Salud (SSA) y diseñada por expertos de la Facultad de Psicología y el Instituto Nacional de Psiquiatría Ramón de la Fuente Muñiz, (UNAM, 2020). Por su parte, el Instituto Politécnico Nacional (IPN) activó una línea telefónica, la Línea de Apoyo Psicológico por COVID-19, (IPN, 2020). Dentro de este contexto, en la Universidad La Salle México, de manera consciente, adaptaron 17 proyectos semestrales, que nacieron originalmente de forma presencial y fueron adecuados hacia los problemas de salud mental que impactan a las personas durante la contingencia sanitaria por COVID-19, atendidos por estudiantes y docentes de las licenciaturas en Psicología, Ciencias de la Educación, Filosofía y Ciencias Religiosas, para brindar información y apoyo a personas que lo requieran, (Universidad La Salle, 2020). Con los valores propios de la filosofía lasallista y sus intervenciones, los estudiantes tienen la oportunidad de aplicar lo aprendido en su formación académica, y adquirir experiencia con situaciones reales.

Conviene distinguir de manera sobresaliente, la labor profesional que al respecto puso en marcha la Universidad La Salle Pachuca a través de Rizoma, (Universidad La Salle Pachuca, 2020), es un equipo de trabajo de psicoterapeutas que brinda de manera gratuita a la comuni- de primeros auxilios psicológicos, contención y acompañamiento emocional e intervención en crisis, de manera telefónica y virtual. Esta información es dada a conocer a través de la página de la universidad, sus redes sociales, docentes y tutores, así como en la Facultad de Ciencias Humanas. De igual modo, el Instituto Mexicano del Seguro Social (IMSS), implementó el Plan de Acción en Salud Mental, ante la emergencia por COVID-19, con una línea de orientación médica telefónica, que atiende a derechohabientes y trabajadores del personal de salud, en donde personal del IMSS en psiquiatría y psicología brinda acompañamiento, técnicas de relajación y psicoeducación para intervenir en momentos de crisis, (IMSS, 2020).

Y, al mismo tiempo que ocurrió en el panorama de la atención pública, muchas otras ofertas surgieron por el lado de la atención psicológica particular, a través de las redes sociales y los buscadores de internet fue posible encontrarse con un gran mercado de consultorios virtuales. Es importante considerar que algunas de estas propuestas aclaran que una de las condiciones para que pueda recibirse la atención psicológica, es no padecer una enfermedad psiquiátrica que requiera internamiento.

Otorgar el valor que merece la salud mental, admite que iniciar o continuar tratamientos psicológicos, permitirá que las consecuencias por el confinamiento y distanciamiento 
social no sean tan adversas para los usuarios, pacientes o clientes de estos servicios. De ahí la importancia y aspiración de este instrumento; existen ciertos lineamientos a los que el profesional de la salud mental debe apegarse para llevar a cabo una adecuada intervención a distancia.

\section{NORMAS MEXICANAS}

Las Normas Oficiales Mexicanas (NOM), -publicadas en el Diario Oficial de la Federación (2010a)-, son regulaciones técnicas que deben cumplirse de manera obligatoria, en el caso de la atención psicológica, son expedidas por la Secretaría de Salud y tienen como propósito establecer las características que deben cumplir los procesos o servicios en los que interviene la salud de las personas. Los psicólogos debemos trabajar con la NOM-004-SSA3-2012 del expediente clínico que tiene como propósito:

[...] establecer con precisión los criterios científicos, éticos, tecnológicos y administrativos obligatorios en la elaboración, integración, uso, manejo, archivo, conservación, propiedad, titularidad y confidencialidad del expediente clínico, el cual se constituye en una herramienta de uso obligatorio para el personal del área de la salud, de los sectores público, social y privado que integran el Sistema Nacional de Salud. (p. 4)
El cumplimiento de esta norma, integra la información y datos personales de un paciente, en el expediente clínico se registra y se refleja el proceso de la atención otorgada y la manera en que se ha realizado la intervención, de igual manera se describe su estado de salud y los pronósticos a su tratamiento, además, si es necesaria, alguna referencia a otro especialista, así como el fin de la psicoterapia. Un expediente completo, debe incluir una ficha de identificación, que contenga los datos generales del usuario y no debe faltar un número de emergencia.

Habría que mencionar otra norma que hay que acatar y se ajusta para la atención en línea, es la NOM-024-SSA3-2010 del expediente clínico electrónico de los sistemas de información de registro electrónico para la salud. En ella se establecen los objetivos y funciones que deberán contener los expedientes electrónicos en salud. Con la intención de que a través del uso de las tecnologías se tenga un mismo lenguaje en el contenido del expediente electrónico, asegurando de igual manera la confidencialidad y seguridad de la información.

\section{AVISO DE PRIVACIDAD}

Para los fines de este argumento, en julio de 2010 se publicó la Ley Federal de Protección de Datos Personales en Posesión de Particulares, a través del Instituto Nacional de Transparencia (INAI), se generó normatividad de carác- 


\section{CONSENTIMIENTO INFORMADO}

ter obligatorio cuya esencia es regular, controlar y comunicar el tratamiento que se le da a la información que las personas proporcionan, de manera que se garantice su privacidad y sus derechos en el manejo de esta; generando guías para el sector privado y público.

El INAI (2013) también da a conocer qué tipo de información se consideran datos personales sensibles:

[...] son aquellos datos personales que afectan a la esfera más íntima de su titular, o cuya utilización indebida pueda dar origen a discriminación o conlleve un riesgo grave para él, como, por ejemplo: su origen racial o étnico; estado de salud pasado, presente y futuro; información genética; creencias religiosas, filosóficas y morales; afiliación sindical; opiniones políticas; preferencia sexual, entre otros. (p. 2)

Por lo expuesto en este apartado, es también una obligación para el psicólogo o psicoterapeuta, generar un Aviso de Privacidad, en el que, a través de un documento físico y electrónico, el responsable de ofrecer el servicio informa al titular que lo recibe, las características principales del tratamiento al que serán sometidos sus datos personales. Mismo que también debe ser firmado por quien acepta el servicio.
El consentimiento informado nos habla de la importancia y la transparencia que debe existir en la relación entre el psicoterapeuta y el paciente, o bien el responsable del paciente si se trata de un menor de edad; recordando que la intervención psicológica es un trabajo y a la vez un proceso de acompañamiento.

Las normas de salud NOM-004SSA3-2012 y NOM-024-SSA3-2010, notificadas en el Diario Oficial de la Federación (2010 a y b) lo incluyen como parte obligatoria del expediente clínico, definiendo así al consentimiento informado:

[...] los documentos escritos, signados por el paciente o su representante legal o familiar más cercano en vínculo, mediante los cuales se acepta un procedimiento médico o quirúrgico con fines diagnósticos, terapéuticos, rehabilitatorios, paliativos o de investigación, una vez que se ha recibido información de los riesgos y beneficios esperados para el paciente. (p.11)

Es por lo que se trata también de una obligación el firmar un consentimiento bajo información para poder intervenir; a través de este documento el paciente conoce -y proporciona evidencia para el profesional de que el usuario está al tanto- cuáles son los procedimientos que se van a llevar a cabo en su tratamiento, cuánto durará dicha atención, los beneficios que obtendrá y en su caso, los posi- 
bles efectos adversos que ocurrieran durante la terapia. Todo ello deberá quedar plasmado con una firma que autoriza nuestra intervención a través de medios físicos y electrónicos

\section{LINEAMIENTOS}

La Asociación Americana de Psicología (American Psychological Association o APA por sus siglas en inglés) es una organización de psicólogos estadounidenses, cuya función es el desarrollo de la psicología de manera profesional y científica, se ha encargado de regir y determinar gran parte del quehacer psicológico internacional desde 1892.

De esta manera, la APA (2013) marca las siguientes directrices a manera de sugerencias o recomendaciones para la práctica de la telepsicología con la finalidad de orientar y educar a los psicólogos que utilizan la tecnología en sus prácticas:

- La telepsicología se define, como la prestación de servicios psicológicos utilizando tecnologías de telecomunicaciones, en las que se incluyen, a través del uso de la computadora o dispositivos móviles, la videollamada o plataformas virtuales para videoconferencia. Tomando en cuenta los posibles beneficios y limitaciones con cada cliente y situación en particular.

- Los psicólogos tienen la obligación ética primaria de proporcionar servicios profesionales solo dentro de los límites de su competencia en función de su educación, formación, experiencia supervisada, consulta, estudio o experiencia profesional.

- Si un cliente/paciente experimenta crisis/emergencia de manera recurrente que sugieran que los servicios en persona pueden ser apropiados, los psicólogos toman medidas razonables para derivar a un cliente/paciente a un recurso de salud mental local o comenzar a brindar servicios en persona.

- Los psicólogos se esfuerzan por obtener y documentar el consentimiento informado que aborde específicamente las inquietudes únicas relacionadas con los servicios de telepsicología que brindan. Al hacerlo, los psicólogos conocen las leyes y regulaciones aplicables, así como los requisitos organizacionales que rigen el consentimiento informado en esta área.

- Los psicólogos que brindan servicios de telepsicología hacen un esfuerzo razonable para proteger y mantener la confidencialidad de los datos y la información relacionados con sus clientes/pacientes e informarles de los riesgos potencialmente mayores de pérdida de confidencialidad inherentes al uso de las tecnologías de telecomunicaciones, si las hubiera. 
- Los psicólogos que brindan servicios de telepsicología toman medidas razonables para garantizar que existan medidas de seguridad para proteger los datos y la información relacionada con sus clientes/pacientes contra el acceso o la divulgación no intencionados.

- Se alienta a los psicólogos a familiarizarse y cumplir con todas las leyes y regulaciones relevantes cuando brinden servicios de telepsicología a clientes/pacientes a través de fronteras jurisdiccionales e internacionales.

Consideremos que, estas pautas no son obligatorias sino aspiracionales, si así lo pusiéramos en práctica los expertos en atención psicológica, brindaríamos servicios apegados a un modelo de calidad, y así mismo lo recibirían consultantes; debido a que estas directrices están formuladas desde la ética y la legalidad de la práctica profesional.

\section{CONDICIONES DE RIESGO QUE NO SE PUEDEN ATENDER EN LÍNEA}

Es posible, por lo tanto, definir que los psicólogos que ofrecemos servicios apegados a un código de ética, sabemos que la protección de los usuarios es el fin último al que se aspira en el quehacer profesional, conscientes de que existen límites en la competencia laboral y en la intervención, de los cuales tenemos que des- lindarnos y derivar o canalizar a los pacientes con el especialista adecuado. La experiencia desde la formación en psicoterapia, nos indica que existen circunstancias que no pueden ser atendidas en línea, debido a que ponen en riesgo la vida del paciente o de otras personas. Estas condiciones son:

- Alteraciones de la senso-percepción

- Ideación, planeación, intento suicida u homicida

- Lesiones autoinfligidas graves

Es imprescindible que los psicólogos tengan en cuenta, que en la práctica clínica y de la salud nos debemos apoyar en las herramientas de clasificación, en las que encontramos los criterios diagnósticos que nos ayudan a realizar las derivaciones de manera más objetiva; para ello nos auxiliamos con el Manual Diagnóstico y Estadístico de Trastornos Mentales (DSM), así como en la Clasificación Estadística Internacional de Enfermedades y Problemas Relacionados con la Salud, (CIE). Y, con ello, es posible darles una dirección adecuada a los usuarios con estas situaciones en las que puede existir riesgo y ofrecerles una resolución apropiada.

Ante cualquiera de las circunstancias mencionadas anteriormente, es primordial derivar al paciente con el especialista psiquiatra, e indicárselo desde el primer contacto, si estas situaciones se presentan ya iniciada la terapia, deben quedar asentadas dentro del expediente clínico junto a su hoja de referencia, en la que 
también se estipula la obligatoria vigilancia del paciente, hasta que El con este pueda asistir a consulta con el médico psiquiatra. En la hoja de referencia también se esclarece la responsabilidad de la que se deslinda el psicólogo, de no ser atendidas las indicaciones. Si el médico psiquiatra determina una terapia en conjunto psiquiatra-psicólogo, debe el paciente regresar con su hoja de contrarreferencia emitida por el médico psiquiatra, solo así podrá llevarse a cabo nuestra intervención.

De la misma manera, según la APA (2013), otras situaciones en las que también se ve limitado el trabajo del psicoterapeuta en línea son cuando el paciente presenta:

- Consumo de drogas. A menos que contemos con una especialización en atención a las adicciones. En estos casos los usuarios deben ser evaluados rigurosamente por expertos capacitados en consumo de drogas. Quién a través de evaluaciones especializadas determinarán si requiere de tratamiento ambulatorio o internamiento.

- Violencia. Ante esta situación, es indispensable que los usuarios acudan a las instancias oficiales de gobierno. Es necesario aclarar que la violencia en sus diferentes modalidades se trata de un delito y requiere ser atendido con los protocolos adecuados, por ello, se hace obligatorio derivarles a centros espe- cializados de atención a la violencia, quienes pueden disponer sus procedimientos e infraestructura para brindar una atención integral.

Igualmente, deben quedar asentadas estas derivaciones en el expediente clínico, junto a su hoja de referencia o bien realizarlas desde el primer contacto con el cliente, sin llevar a cabo la consulta.

\section{CONDICIONES MATERIALES}

Recordando que debido a la contingencia por COVID-19, la psicoterapia tuvo que trasladarse a los espacios en línea, existen también condiciones materiales necesarias para para llevarse a cabo, es básico para ello contar con:

- Una línea telefónica en la que se realice el primer contacto paciente-psicólogo. Por medio de ella también se proporciona la información del procedimiento de la psicoterapia en línea y se determina si el usuario es candidato, o no, a la intervención a distancia.

- La confirmación del pago por los servicios (puede ser también a través de medios electrónicos como una transferencia bancaria).

- Los formatos electrónicos que conforman el 
expediente clínico electrónico.

- Equipo de cómputo o dispositivo móvil que cuente con cámara, micrófono, bocinas y navegador.

- Conexión a internet estable.

- Una plataforma de videoconferencia que cuente con aviso de privacidad (deseable).

- Cuidar en extremo la confidencialidad de la información y de la sesión.

- Un espacio sin interrupciones.

- Una evaluación de la calidad de la sesión (deseable).

De contar con estos elementos, estaríamos hablando de una situación ideal para abordar esta modalidad de psicoterapia.

\section{CONCLUSIÓN}

La situación generada por la pandemia de COVID-19 generó modificaciones en la práctica profesional de la atención psicológica, trasladando el consultorio a una modalidad virtual. Los profesionales de la salud mental nos adap- tamos a este contexto, con la intención de que el paciente siga contando con la atención profesional que necesita y merece. Para esta fecha, llevamos más de un año en confinamiento, no sabemos de manera certera cuándo terminará, y si la psicoterapia en línea será parte de la llamada «nueva normalidad», en donde gracias a las tecnologías es posible la práctica profesional.

Es básico que, como profesionales de la salud, ofertemos servicios que cumplan todas las normas y regulaciones oficiales que informen y protejan los derechos de los usuarios. Reconociendo, si es el caso, los límites de nuestro alcance en la práctica.

Cabe mencionar que los sistemas públicos de atención psicológica limitaron su atención presencial exclusivamente a emergencias, con las medidas extremas de sana distancia para evitar contagios, cancelando los servicios regulares. Es en este espacio, donde oportunamente brinda sus servicios la atención psicológica en línea.

De lo anterior cabe mencionar que, la situación ideal para abordar la psicoterapia a distancia requiere tener cubiertos los elementos antes descritos de una manera ordenada en su manejo. Sirva este documento de guía para una atención psicológica y psicoterapéutica experta, en modalidad virtual, con la intención de que estas pautas conduzcan hacia una práctica ética y competitiva, independientemente del enfoque terapéutico desde el que se aborde la intervención. 
American Psychiatric Association. (2014). Manual diagnóstico y estadístico de los trastornos mentales DSM-5 (5a. ed.). Editorial Médica Panamericana.

American Psychological Association. (2013, julio 31). Directrices para la práctica de la telepsicología. [Publicación digital] https://www.apa.org/pubs/journals/features/amp-a0035001.pdf

Diario Oficial de la Federación. (04 de marzo de 2010b). NOM-024-SSA3-2010 del expediente clínico electrónico. Diario oficial de la federación. México. http://www.dof. gob.mx/normasOficiales/4151/salud/salud.htm

Diario Oficial de la Federación. (05 de octubre de 2010a). NOM-004-SSA3-2012 del expediente clínico. Diario oficial de la federación. México. http://dof.gob.mx/nota_ detalle_popup.php?codigo $=5272787$

Instituto Mexicano del Seguro Social. (2020). Por la emergencia sanitaria COVID-19, el IMSS ha atendido mil 379 llamadas para la atención en salud mental. [Mensaje en un blog] Prensa IMSS. http://www.imss.gob.mx/prensa/archivo/202006/394

Instituto Nacional de transparencia y Acceso a la Información. (2013). El abc del aviso de privacidad. [Publicación digital] México. http://abcavisosprivacidad.ifai.org.mx/ PDF/El\%20ABC\%20del\%20Aviso\%20 de\%20Privacidad.pdf

Instituto Politécnico Nacional. (2020). Línea de apoyo psicológico por COVID-19. [Mensaje en un blog]. Gobierno de México. https://www.ipn.mx/imageninstitu- cional/comunicados/ver-comunicado. html?y=2020\&n=76

Organización Mundial de la Salud, (2018). Clasificación Internacional de Enfermedades y Problemas Relacionados con la Salud (CIE11). Onceava Revisión. Suiza.

Secretaría de Salud. (2020). Lineamiento para la metodología de cálculo del semáforo de riesgo epidémico COVID-19. [Publicación digital] México.https://coronavirus. gob.mx/wp-content/uploads/2020/10/ SemaforoCOVID_Metodo.pdf

Sociedad Mexicana de Psicología. (2010). Código ético del psicólogo. México.

Universidad Nacional Autónoma de México. (2020). Bienvenida(o) al cuestionario para la detección de riesgos a la salud mental COVID-19. [Página web] Secretaría de Salud. https://misalud.unam.mx/ COVID19/

Universidad La Salle. (2020, junio 4). Conoce estos 17 proyectos lasallistas en apoyo a la salud mental durante la contingencia. [Mensaje en un blog] Hoy en La Salle. https://hoy.lasalle.mx/conoce-estos-17-proyectos-lasallistas-en-apoyo-a-la-salud-mental-durante-la-contingencial

Universidad La Salle Pachuca. (2020, agosto 20). Rizoma. [Mensaje en un blog] $\mathrm{Co}^{-}$ munidad La Salle Pachuca. http://www. lasallep.edu.mx/noticias/Comunidad/ rizoma.html 